\title{
Novel thiophene derivatives with sulfonamide, isoxazole, benzothiazole, quinoline and anthracene moieties as potential anticancer agents
}

\author{
MOSTAFA M. GHORAB ${ }^{1,2 *}$ \\ MAHMOUD S. BASHANDY $Y^{3,4}$ \\ MANSOUR S. ALSAID ${ }^{1}$ \\ ${ }^{1}$ Department of Pharmacognosy \\ College of Pharmacy, King Saud \\ University, P.O. Box 2457, Riyadh \\ 11451, Kingdom of Saudi Arabia \\ ${ }^{2}$ Department of Drug Radiation \\ Research, National Center for \\ Radiation Research and Technology \\ Atomic Energy, Authority, P.O.Box 29 \\ Nasr City, Cairo, Egypt \\ ${ }^{3}$ Department of Chemistry, Faculty of \\ Science (Boy's), Al-Azhar University \\ Nasr City, Cairo, Egypt \\ ${ }^{4}$ Department of Chemistry, University \\ College at Al-Jomoom, Umm Al-Qura \\ University, 2026, Makkah \\ Saudi Arabia
}

\begin{abstract}
A novel series of thiophenes having biologically active sulfonamide 2-11, 3-methylisoxazole 12, 4-methoxybenzo[d] thiazole 13, quinoline 14, 15, benzoylphenylamino 16, and anthracene-9,10-dione $\mathbf{1 7}$ moieties were prepared. Structures of the newly synthesized compounds were established by elemental analysis and spectral data. All newly synthesized compounds were evaluated for their in vitro anticancer activity against human breast cancer cell line (MCF7). Most of the screened compounds showed cytotoxic activities compared to doxorubicin as a positive control. Compounds 6, 7, 9 and 13 (IC ${ }_{50}$ values $10.25,9.70,9.55$ and $9.39 \mu \mathrm{mol} \mathrm{L}^{-1}$ ) revealed higher cytotoxic activities than that of doxorubicin $\left(I C_{50}=32.00 \mu \mathrm{mol} \mathrm{L}^{-1}\right)$. Also, compounds 5,8 and 10 were found nearly as active as doxorubicin $\left(I C_{50} 28.85,23.48\right.$ and $\left.27.51 \mu \mathrm{mol} \mathrm{L}{ }^{-1}\right)$.
\end{abstract}

Keywords: thiophenes, sulfonamides, isoxazole, benzothiazole, quinoline, anthracene, anticancer activity

Accepted July 3, 2014

Thiophenes have been reported to possess interesting biological and pharmacological activities where several derivatives are used as antibacterial (1-3), anti-inflammatory (4), anticancer $(5,6)$, and antiviral agents (7). Also, sulfonamides constitute an important class of drugs, with several types of pharmacological agents possessing anticancer activity (812), among others. A large number of structurally novel sulfonamides have ultimately been reported to show substantial anticancer activity in vitro and in vivo (13). Several mechanisms have been reported for anticancer activity of sulfonamide compounds and the most prominent of these mechanisms was thought to be the inhibition of carbonic anhydrase (14-16). The mechanism of tumor inhibition by the sulfonamide carbonic anhydrase

*Correspondence; e-mail: mmsghorab@yahoo.com 

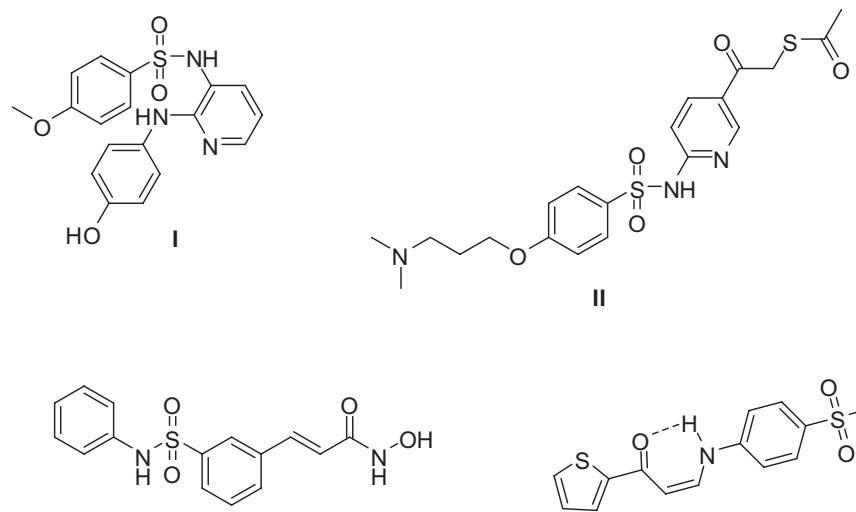

III

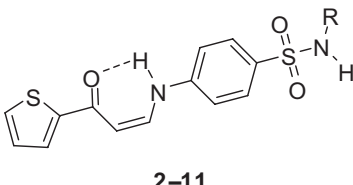

$2-11$

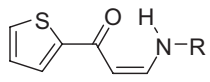

$12-17$

Fig. 1. Biologically active compounds I-III and structures of target compounds $\mathbf{2 - 1 7 . ~}$

inhibitor was suggested by Chegwidden and Spencer (17). These compounds may reduce the provision of bicarbonate for the synthesis of nucleotides and other cell components such as membrane lipids. Also, isoxazole, benzothiazole, quinoline and anthracene derivatives posseses a wide range of biological activities, including anti-inflammatory (18), antibacterial (19) and anticancer activity (20-22). In addition, several novel quinoline derivatives have been reported to show substantial anticancer activities (23-26). Also, a host of structurally novel sulfonamides have recently been reported to show substantial anticancer activity in vitro and/or in vivo. Since the discovery of E-7010 (I , 27), sulfonamides have emerged as an important class of anticancer agents, which interact with a wide range of different cellular targets such as disruption of microtubules assembly and carbonic anhydrase inhibition (17). Some of these compounds having the $\mathrm{NH}$ of the sulfonamide group substituted by an aryl group showed potent anticancer activity such as KD5170 (II) and PXD101 (III) (28). In continuation of our work (29-33), it seemed of interest to design and synthesize a novel series of thiophenes bearing biologically active sulfonamide, isoxazole, benzoxazole, quinoline and anthracene moieties and to evaluate their anticancer activity.

\section{EXPERIMENTAL}

Melting points (uncorrected) were determined in open capillaries on a Gallenkemp melting point apparatus (Sanyo Gallenkemp, UK). Pre-coated silica gel plates (silica gel 0.25 mm, 60 G F 254, Merck, Germany) were used for thin layer chromatography. Dichloromethane/methanol $(9.5: 0.5, V / V)$ mixture was used as a developing solvent system. IR spectra were recorded in $\mathrm{KBr}$ discs using an IR-470 Shimadzu spectrometer (Shimadzu, 
Japan). NMR spectra in DMSO- $d_{6}$ were recorded on Bruker Ac-500 ultra shield NMR spectrometer ( $\delta$ in ppm, Bruker, Switzerland) at $500 \mathrm{MHz}$, using TMS as an internal standard. Elemental analyses were performed on a Carlo Erba 1108 Elemental Analyzer (Heraeus, Germany). Elemental analyses of all compounds were within $\pm 0.4 \%$ of the theoretical values. Starting compound, 1-(thiophen-2-yl)ethanone was purchased from Sigma (USA).

\section{Syntheses}

3-(Dimethylamino)-1-(thiophen-2-yl)prop-2-en-1-one (1) was prepared according to the reported method (34).

(Z)-4-(3-Oxo-3-(thiophen-2-yl)prop-1-enylamino)benzenesulfonamide (2), (Z)-N-carbamimidoyl-4-(3-oxo-3-(thiophen-2-yl)prop-1-enylamino)benzenesulfonamide (3), (Z)-N-(3-methylisoxazol-5-yl)-4-(3-oxo-3-(thiophen-2-yl)prop-1-enylamino)benzenesulfonamide (4), (Z)-N-(3,4-dimethyl-isoxazol-5-yl)-4-(3-oxo-3-(thiophen-2-yl)prop-1-enylamino)benzene-sulfonamide (5), (Z)-4-(3-oxo-3-(thiophen-2-yl)prop-1-enylamino)-N-(thiazol-2-yl)benzenesulfonamide (6), (Z)-4(3-oxo-3-(thiophen-2-yl)prop-1-enylamino)-N-(1-phenyl-1H-pyrazol-5-yl)benzenesulfonamide (7), (Z)-4-(3-oxo-3-(thiophen-2-yl)prop-1-enylamino)-N-(pyridin-2-yl)benzenesulfonamide (8), (Z)-4(3-oxo-3-(thiophen-2-yl)prop-1-enylamino)-N-(pyrimidin-2-yl)benzenesulfonamide (9), (Z)-N-(4methylpyrimidin-2-yl)-4-(3-oxo-3-(thiophen-2-yl)prop-1-enylamino)benzenesulfonamide (10) and (Z)-N-(4,6-dimethylpyrimidin-2-yl)-4-(3-oxo-3-(thiophen-2-yl)prop-1-enylamino)benzenesulfonamide (11). General procedure. - A mixture of (1) $(1.81 \mathrm{~g}, 0.01 \mathrm{~mol})$ and sulfonamide derivatives $(0.01 \mathrm{~mol})$ in ethanol $(20 \mathrm{~mL})$ containing acetic acid $(10 \mathrm{~mL})$ was refluxed for $6 \mathrm{~h}$. The obtained solid was recrystallized from dioxane while hot to give 2-11.

(Z)-3-(3-Methylisoxazol-5-ylamino)-1-(thiophen-2-yl)prop-2-en-1-one (12). - A mixture of $1(1.81 \mathrm{~g}, 0.01 \mathrm{~mol})$ and 3-methyl-5-aminoisoxazole $(0.98 \mathrm{~g}, 0.01 \mathrm{~mol})$ in ethanol $(20 \mathrm{~mL})$, containing acetic acid $(10 \mathrm{~mL})$ was refluxed for $6 \mathrm{~h}$. The obtained solid was recrystallized from dioxane to give $\mathbf{1 2 .}$

(Z)-3-(4-Methoxybenzo[d]thiazol-2-ylamino)-1-(thiophen-2-yl)prop-2-en-1-one (13). - A mixture of $(\mathbf{1})(1.81 \mathrm{~g} ; 0.01 \mathrm{~mol})$ and 2-amino-4-methoxybenzothiazole $(1.80 \mathrm{~g}, 0.01 \mathrm{~mol})$ in ethanol $(20 \mathrm{~mL})$ containing $(10 \mathrm{~mL})$ acetic acid was refluxed for $8 \mathrm{~h}$. The obtained solid was recrystallized from ethanol to give $\mathbf{1 3}$.

(Z)-3-(Quinolin-3-ylamino)-1-(thiophen-2-yl)prop-2-en-1-one (14) and (Z)-3-(2-methylquinolin-4-ylamino)-1-(thiophen-2-yl)prop-2-en-1-one (15). - A mixture of $\mathbf{1}(1.81 \mathrm{~g}, 0.01 \mathrm{~mol})$ and 3 -aminoquinoline or 4-amino-2-methylquinoline $(0.01 \mathrm{~mol})$ in absolute ethanol $(20 \mathrm{~mL})$ glacial acetic acid $(10 \mathrm{~mL})$ was refluxed for $18 \mathrm{~h}$. The obtained solid while hot was recrystallized from dioxane to give $\mathbf{1 4}$ and $\mathbf{1 5}$, respectively.

(Z)-3-(4-Benzoylphenylamino)-1-(thiophen-2-yl)prop-2-en-1-one (16) and (Z)-2-(3-oxo-3(thiophen-2-yl)prop-1-enylamino)anthracene-9,10-dione (17). - A mixture of 1 (1.81 g, 0.01 $\mathrm{mol}$ ) and 4-aminophenyl-phenyl-methanone or 2-aminoanthracene-9,10-dione (0.01 mol) in ethanol $(20 \mathrm{~mL})$, containing acetic acid $(10 \mathrm{~mL})$ was refluxed for $8 \mathrm{~h}$. The solid obtained, while hot, was recrystallized from dioxane to give (16) and (17), respectively. Physical, chemical and spectral data of newly synthesized compounds are given in Tables I and II. 
M. M. Ghorab et al.: Novel thiophene derivatives with sulfonamide, isoxazole, benzothiazole, quinoline and anthracene moieties as potential anticancer agents, Acta Pharm. 64 (2014) 419-431.

Table I. Physical and analytical data of the newly synthesized compounds

\begin{tabular}{|c|c|c|c|c|c|c|}
\hline \multirow{2}{*}{ Compd. } & \multirow{2}{*}{ M. p. $\left({ }^{\circ} \mathrm{C}\right)$} & \multirow{2}{*}{ Yield (\%) } & \multirow{2}{*}{$\begin{array}{l}\text { Mol. formula } \\
\qquad\left(M_{\mathrm{r}}\right)\end{array}$} & \multicolumn{3}{|c|}{ Analysis calcd./found (\%) } \\
\hline & & & & C & $\mathrm{H}$ & $\mathrm{N}$ \\
\hline \multirow{2}{*}{2} & \multirow{2}{*}{254.6} & \multirow{2}{*}{93} & \multirow{2}{*}{$\begin{array}{c}\mathrm{C}_{13} \mathrm{H}_{12} \mathrm{~N}_{2} \mathrm{O}_{3} \mathrm{~S}_{2} \\
(308.38)\end{array}$} & 50.63 & 3.92 & 9.08 \\
\hline & & & & 50.33 & 3.62 & 9.31 \\
\hline \multirow{2}{*}{3} & \multirow{2}{*}{265.2} & \multirow{2}{*}{90} & \multirow{2}{*}{$\begin{array}{c}\mathrm{C}_{14} \mathrm{H}_{14} \mathrm{~N}_{4} \mathrm{O}_{3} \mathrm{~S}_{2} \\
(350.42)\end{array}$} & 47.99 & 4.03 & 15.99 \\
\hline & & & & 47.60 & 4.28 & 15.68 \\
\hline \multirow{2}{*}{4} & \multirow{2}{*}{239.8} & \multirow{2}{*}{89} & \multirow{2}{*}{$\begin{array}{c}\mathrm{C}_{17} \mathrm{H}_{15} \mathrm{~N}_{3} \mathrm{O}_{4} \mathrm{~S}_{2} \\
(389.45)\end{array}$} & 52.43 & 3.88 & 10.79 \\
\hline & & & & 52.13 & 3.57 & 10.43 \\
\hline \multirow{2}{*}{5} & \multirow{2}{*}{238.1} & \multirow{2}{*}{86} & \multirow{2}{*}{$\begin{array}{c}\mathrm{C}_{18} \mathrm{H}_{17} \mathrm{~N}_{3} \mathrm{O}_{4} \mathrm{~S}_{2} \\
(403.48)\end{array}$} & 53.58 & 4.25 & 10.41 \\
\hline & & & & 53.23 & 4.55 & 10.08 \\
\hline \multirow{2}{*}{6} & \multirow{2}{*}{269.8} & \multirow{2}{*}{79} & \multirow{2}{*}{$\begin{array}{c}\mathrm{C}_{16} \mathrm{H}_{13} \mathrm{~N}_{3} \mathrm{O}_{3} \mathrm{~S}_{3} \\
(391.49)\end{array}$} & 49.09 & 3.35 & 10.73 \\
\hline & & & & 49.33 & 3.71 & 10.42 \\
\hline \multirow{2}{*}{7} & \multirow{2}{*}{242.4} & \multirow{2}{*}{74} & \multirow{2}{*}{$\begin{array}{c}\mathrm{C}_{22} \mathrm{H}_{18} \mathrm{~N}_{4} \mathrm{O}_{3} \mathrm{~S}_{2} \\
(450.53)\end{array}$} & 58.65 & 4.03 & 12.44 \\
\hline & & & & 58.96 & 4.29 & 12.70 \\
\hline 8 & 1930 & 80 & $\mathrm{C}_{18} \mathrm{H}_{15} \mathrm{~N}_{3} \mathrm{O}_{3} \mathrm{~S}_{2}$ & 56.09 & 3.92 & 10.90 \\
\hline 0 & 190.0 & 00 & $(385.46)$ & 56.33 & 3.71 & 10.58 \\
\hline 9 & 3017 & 78 & $\mathrm{C}_{17} \mathrm{H}_{14} \mathrm{~N}_{4} \mathrm{O}_{3} \mathrm{~S}_{2}$ & 52.84 & 3.65 & 14.50 \\
\hline & & & $(386.45)$ & 52.49 & 3.31 & 14.77 \\
\hline 10 & 2769 & 77 & $\mathrm{C}_{18} \mathrm{H}_{16} \mathrm{~N}_{4} \mathrm{O}_{3} \mathrm{~S}_{2}$ & 53.98 & 4.03 & 13.99 \\
\hline 10 & 270.9 & 71 & $(400.47)$ & 53.62 & 4.29 & 13.69 \\
\hline 11 & 2626 & 83 & $\mathrm{C}_{19} \mathrm{H}_{18} \mathrm{~N}_{4} \mathrm{O}_{3} \mathrm{~S}_{2}$ & 55.05 & 4.38 & 13.52 \\
\hline & & & $(414.50)$ & 55.32 & 4.09 & 13.84 \\
\hline 12 & 1472 & 61 & $\mathrm{C}_{11} \mathrm{H}_{10} \mathrm{~N}_{2} \mathrm{O}_{2} \mathrm{~S}$ & 56.39 & 4.30 & 11.96 \\
\hline & & & $(234.27)$ & 56.77 & 4.65 & 11.86 \\
\hline 13 & 1308 & 71 & $\mathrm{C}_{15} \mathrm{H}_{12} \mathrm{~N}_{2} \mathrm{O}_{2} \mathrm{~S}_{2}$ & 56.94 & 3.82 & 8.85 \\
\hline & & 11 & (316.39) & 56.69 & 3.58 & 8.49 \\
\hline 14 & 2158 & 66 & $\mathrm{C}_{16} \mathrm{H}_{12} \mathrm{~N}_{2} \mathrm{OS}$ & 68.55 & 4.31 & 9.99 \\
\hline 14 & & 60 & $(280.34)$ & 68.91 & 4.08 & 10.12 \\
\hline 15 & 208.8 & 69 & $\mathrm{C}_{17} \mathrm{H}_{14} \mathrm{~N}_{2} \mathrm{OS}$ & 69.36 & 4.79 & 9.52 \\
\hline & & & (294.37) & 69.70 & 4.46 & 9.19 \\
\hline 16 & 1689 & 61 & $\mathrm{C}_{20} \mathrm{H}_{15} \mathrm{NO}_{2} \mathrm{~S}$ & 72.05 & 4.53 & 4.20 \\
\hline & & 01 & (333.40) & 72.33 & 4.19 & 4.52 \\
\hline 17 & 2118 & 77 & $\mathrm{C}_{21} \mathrm{H}_{13} \mathrm{NO}_{3} \mathrm{~S}$ & 70.18 & 3.65 & 3.90 \\
\hline 17 & 211.8 & 71 & $(359.06)$ & 70.51 & 3.19 & 3.58 \\
\hline
\end{tabular}


M. M. Ghorab et al:: Novel thiophene derivatives with sulfonamide, isoxazole, benzothiazole, quinoline and anthracene moieties as potential anticancer agents, Acta Pharm. 64 (2014) 419-431.

Table II. Spectral data of the newly synthesized compounds

\begin{tabular}{|c|c|c|c|}
\hline Compd. & $\operatorname{IR}\left(v, \mathrm{~cm}^{-1}\right)$ & $\begin{array}{l}{ }^{1} \mathrm{H} \text { NMR }\left(\mathrm{DMSO}-d_{6}\right) \\
(\delta, \mathrm{ppm})\end{array}$ & $\begin{array}{l}{ }^{13} \mathrm{C} \text { NMR }\left(\mathrm{DMSO}-d_{6}\right) \\
(\delta, \mathrm{ppm})\end{array}$ \\
\hline 2 & $\begin{array}{l}3340,3248,3190\left(\mathrm{NH}, \mathrm{NH}_{2}\right) \\
3101(\mathrm{CH} \text { arom. }), 1628 \\
(\mathrm{C}=\mathrm{O}), 1360,1157\left(\mathrm{SO}_{2}\right)\end{array}$ & $\begin{array}{l}\text { 6.1, } 7.1(2 \mathrm{~d}, 2 \mathrm{H}, \mathrm{CH}=\mathrm{CH}, J=7.1, \\
7.6 \mathrm{~Hz}), 7.2-8.0(\mathrm{~m}, 9 \mathrm{H}, \mathrm{Ar}-\mathrm{H}+ \\
\left.\mathrm{SO}_{2} \mathrm{NH}_{2}\right), 10.4\left(\mathrm{~s}, 1 \mathrm{H}, \mathrm{NH}, \mathrm{D}_{2} \mathrm{O}\right. \\
\text { exchangeable) }\end{array}$ & $\begin{array}{l}94.8,115.8(2), 128.5(2), \\
128.6,130.4,138.0,142.5, \\
144.2,145.7,146.4,180.3\end{array}$ \\
\hline 3 & $\begin{array}{l}3425,3320,3185\left(\mathrm{NH}, \mathrm{NH}_{2}\right), \\
3100 \text { ( } \mathrm{CH} \text { arom. }), 2970,2897 \\
(\mathrm{CH} \text { aliph.), } 1646(\mathrm{C}=\mathrm{O}), \\
1380,1130\left(\mathrm{SO}_{2}\right)\end{array}$ & $\begin{array}{l}\text { 6.4, } 7.4(2 \mathrm{~d}, 2 \mathrm{H}, \mathrm{CH}=\mathrm{CH}, \mathrm{J}=7.7, \\
7.8 \mathrm{~Hz}), 6.7\left(\mathrm{~s}, 2 \mathrm{H}, \mathrm{NH}_{2}, \mathrm{D}_{2} \mathrm{O}-\mathrm{ex}-\right. \\
\text { changeable), 7.5-7.9 }(\mathrm{m}, 7 \mathrm{H}, \\
\mathrm{Ar}-\mathrm{H}), 8.1(\mathrm{~s}, 1 \mathrm{H}, \mathrm{NH} \text { imino; } \\
\mathrm{D}_{2} \mathrm{O}-\text { exchangeable), } 10.3(\mathrm{~s}, 1 \mathrm{H}, \\
\mathrm{NH} \text { aniline; } \mathrm{D}_{2} \mathrm{O}-\text { exchangeable), } \\
11.7\left(\mathrm{~s}, 1 \mathrm{H}, \mathrm{SO}_{2} \mathrm{NH}, \mathrm{D}_{2} \mathrm{O}-\text { ex- }\right. \\
\text { changeable ) }\end{array}$ & $\begin{array}{l}94.6,115.6(2), 128.4(2), \\
128.6,129.8,137.7,138.6, \\
144.4,145.7,146.5,158.0, \\
180.2\end{array}$ \\
\hline
\end{tabular}

$3464,3370(2 \mathrm{NH}), 3066$ (CH arom.), 2966, 2860 (CH aliph.), 1632 ( $\mathrm{C}=\mathrm{O})$, $1589(\mathrm{C}=\mathrm{N}), 1334,1161\left(\mathrm{SO}_{2}\right)$ $2.3\left(\mathrm{~s}, 3 \mathrm{H}, \mathrm{CH}_{3}\right), 6.1(\mathrm{~d}, 1 \mathrm{H}$, $\mathrm{CHCO} ; J=7.2 \mathrm{~Hz}), 6.4$ (s, 1H, CH 128.6 (2), 128.8, 130.6, oxazole), $7.2(\mathrm{~d}, 1 \mathrm{H}, \underline{\mathrm{CH}}-\mathrm{NH} ; \mathrm{J}=$ 7.5 Hz), 7.3-7.9 (m, 7H, Ar-H), 10.4 (d, 1H, NH; J = 7.4 Hz), 11.2 (s, $1 \mathrm{H}, \mathrm{SO}_{2} \mathrm{NH}, \mathrm{D}_{2} \mathrm{O}$-exchangeable)

3317, 3209 (2NH), 2924, $28602.1\left(\mathrm{~s}, 6 \mathrm{H}, 2 \mathrm{CH}_{3}\right), 6.1$ (d, 1H, (CH aliph.), $1685(\mathrm{C}=\mathrm{O}), \quad \mathrm{CHCO} ; J=7.2 \mathrm{~Hz}), 7.2(\mathrm{~d}, 1 \mathrm{H}$, $1635(\mathrm{C}=\mathrm{N}), 1340,1192\left(\mathrm{SO}_{2}\right)$ $\mathrm{CH}-\mathrm{NH}$; $J=7.4 \mathrm{~Hz}), 7.3-7.9(\mathrm{~m}$, $7 \mathrm{H}, \mathrm{Ar}-\mathrm{H}), 10.5$ (d, 1H, NH; J = $7.8 \mathrm{~Hz}), 11.7$ (s, $1 \mathrm{H}, \mathrm{SO}_{2} \mathrm{NH}$, $\mathrm{D}_{2} \mathrm{O}$-exchangeable)

3390, 3290 (2NH), 3090 (CH arom.), 2940, 2860 ( $\mathrm{CH}$ aliph.), 1635 ( $\mathrm{C}=\mathrm{O})$, $1593(\mathrm{C}=\mathrm{N}), 1373,1138\left(\mathrm{SO}_{2}\right)$

$6.1,7.2(2 \mathrm{~d}, 2 \mathrm{H}, \mathrm{CH}=\mathrm{CH} ; J=7.2$, $7.3 \mathrm{~Hz}), 6.4,7.3$ (2d, 2H, CH=CH thiazole; $J=7.7 \mathrm{~Hz}), 7.4-8.0(\mathrm{~m}$, $7 \mathrm{H}, \mathrm{Ar}-\mathrm{H}), 10.4$ (d, 1H, NH; $J=$ $7.0 \mathrm{~Hz}), 11.7$ (s, 1H, SO $\mathrm{NH}^{\mathrm{NH}}$ $\mathrm{D}_{2} \mathrm{O}$-exchangeable)

3340, 3290 (2NH), 3065 (CH arom.), 2970, 2836 (CH aliph.), 1646 ( $\mathrm{C}=\mathrm{O})$, $1610(\mathrm{C}=\mathrm{N}), 1370,1183\left(\mathrm{SO}_{2}\right)$

6.1, $7.2(2 \mathrm{~d}, 2 \mathrm{H}, \mathrm{CH}=\mathrm{CH}, J=7.6$, $7.7 \mathrm{~Hz}), 6.4,7.8$ (2d, 2H, CH-CH pyrazole, $J=7.1,7.3 \mathrm{~Hz}), 7.3-8.0$ (m, 12H, Ar-H), 10.4 (d, 1H, NH, $J=7.6 \mathrm{~Hz}), 11.7$ (s, 1H, SO $\mathrm{NH}$, $\mathrm{D}_{2} \mathrm{O}$-exchangeable)

3310, 3280 (2NH), 3055

(CH arom.), 2940, 2860
6.4, $7.2(2 \mathrm{~d}, 2 \mathrm{H}, \mathrm{CH}=\mathrm{CH}, J=7.1$, $7.3 \mathrm{~Hz}), 7.3-8.1$ (m, 11H, Ar-H), $10.4(\mathrm{~d}, 1 \mathrm{H}, \mathrm{NH}, J=7.8 \mathrm{~Hz}), 11.7$ $\left(\mathrm{s}, 1 \mathrm{H}, \mathrm{SO}_{2} \mathrm{NH}, \mathrm{D}_{2} \mathrm{O}\right.$-exchangeable)
$5.8,10.3,95.5,100.0,116.0$ (2), 128.7 (2), 130.1, 130.6, 133.5, 142.1, 145.1, 145.6, $146.2,155.5,161.4,183.4$

$94.9,108.1,115.8(2), 128.6$ (2), 129.9, 130.4, 132.6, 133.3, 135.1, 142.4, 144.1, $146.4,170.0,180.3$

$95.4,99.9,116.0(2), 124.2$ (2), 127.4, $128.6(2), 128.7$, 130.6 (2), 131.8, 138.2, $139.5,142.2,143.9,144.0$, $144.9,145.6,146.3,180.4$

95.0, 112.0, 113.2, 115.8 (2), 128.6 (2), 128.8, 129.9, 135.3, 139.9, 140.1, 143.2, $144.2,145.7,146.3,152.8$, 180.4 
$3464,3267(2 \mathrm{NH}), 3035$

(CH arom.), 2939, 2870

9

(CH aliph.), 1655 ( $\mathrm{C}=\mathrm{O})$, $1582(\mathrm{C}=\mathrm{N}), 1338,1195\left(\mathrm{SO}_{2}\right)$

3433, 3370 (2NH), 3060 (CH arom.), 2947, 2862

(CH aliph.), 1632 ( $\mathrm{C}=\mathrm{O})$, $1593(\mathrm{C}=\mathrm{N}), 1363,1153\left(\mathrm{SO}_{2}\right)$
6.4, $7.3(2 \mathrm{~d}, 2 \mathrm{H}, \mathrm{CH}=\mathrm{CH}, J=7.0$, $7.2 \mathrm{~Hz}), 7.4-8.5$ (m, 10H, Ar-H), $10.4(\mathrm{~d}, 1 \mathrm{H}, \mathrm{NH}, J=7.6 \mathrm{~Hz}), 11.7$ (s, $1 \mathrm{H}, \mathrm{SO}_{2} \mathrm{NH}, \mathrm{D}_{2} \mathrm{O}$-exchangeable)

$2.3\left(\mathrm{~s}, 3 \mathrm{H}, \mathrm{CH}_{3}\right), 6.4,7.3(2 \mathrm{~d}, 2 \mathrm{H}$, $\mathrm{CH}=\mathrm{CH}, J=7.1,7.4 \mathrm{~Hz}), 7.4-8.3$ (m, 9H, Ar-H), 10.4 (d, 1H, NH, $J=8.1 \mathrm{~Hz}), 11.7\left(\mathrm{~s}, 1 \mathrm{H}, \mathrm{SO}_{2} \mathrm{NH}\right.$, $\mathrm{D}_{2} \mathrm{O}$-exchangeable)

3210 (2NH), 3066 (CH arom.), 2993, 2816 (CH aliph.), 1697 (C=O), 1635, $1593(2 \mathrm{C}=\mathrm{N}), 1373,1157$ $\left(\mathrm{SO}_{2}\right)$

3132 (NH), 3082 (CH arom.), 2.3 (s, 3H, $\left.\mathrm{CH}_{3}\right), 6.4$ (s, 1H, CH 2940, 2836 (CH aliph.), 1642 isoxazole), 6.2, 7.3 (2d, 2H, $(\mathrm{C}=\mathrm{O}), 1635(\mathrm{C}=\mathrm{N})$

13

$3421(\mathrm{NH}), 3093(\mathrm{CH}$ arom.), 2930, 2860 (CH aliph.), 1645 (C=O), $1635(\mathrm{C}=\mathrm{N})$

3209 (NH), 3047 (CH arom.), 6.2, 6.5 (2d, 2H, CH=CH; J = 7.2, 2920, 2836 (CH aliph.), $16517.3 \mathrm{~Hz}), 7.4-8.0$ (m, 8H, Ar-H), $(\mathrm{C}=\mathrm{O}), 1612(\mathrm{C}=\mathrm{N})$

$3340(\mathrm{NH}), 3078(\mathrm{CH}$ arom.), 2970, 2830 (CH 15 aliph.), 1643 (C=O), $1620(\mathrm{C}=\mathrm{N})$

3433 (NH), 3046 (CH arom.), 2930, 2840 (CH aliph.), $1640(2 \mathrm{C}=\mathrm{O})$

$3444(\mathrm{NH}), 3070(\mathrm{CH}$ arom.), 2960, 2840 (CH (m, 3H, Ar-H), 10.3 (d, 1H, NH, $J=7.5 \mathrm{~Hz}$ ) (m, 6H, Ar-H), 8.5 (d, 1H, NH; $J=6.8 \mathrm{~Hz}$ ) $11.8(\mathrm{~d}, 1 \mathrm{H}, \mathrm{NH}, J=7.5 \mathrm{~Hz})$ (m, 7H, Ar-H), 8.4 (d, 1H, NH; $J=7.8 \mathrm{~Hz}$ ) $6.2,7.3(2 \mathrm{~d}, 2 \mathrm{H}, \mathrm{CH}=\mathrm{CH}, J=7.4$, $7.5 \mathrm{~Hz}), 7.2-8.0$ (m, 12H, Ar-H), $10.5(\mathrm{~d}, 1 \mathrm{H}, \mathrm{NH} ; J=7.1 \mathrm{~Hz})$

$6.5,6.9(2 \mathrm{~d}, 2 \mathrm{H}, \mathrm{CH}=\mathrm{CH} ; J=7.0$, $7.2 \mathrm{~Hz}), 6.9-8.2(\mathrm{~m}, 10 \mathrm{H}, \mathrm{Ar}-\mathrm{H})$, $2.2\left(\mathrm{~s}, 6 \mathrm{H}, 2 \mathrm{CH}_{3}\right), 6.4,7.2(2 \mathrm{~d}, 2 \mathrm{H}, 23.0(2), 95.1,99.6,115.2(2)$, $\mathrm{CH}=\mathrm{CH}, \mathrm{J}=7.3,7.5 \mathrm{~Hz}), 6.7-7.9 \quad 128.4$ (2), 128.6, 129.9, 133.3, (m, 8H, Ar-H), 10.4 (d, 1H, NH, 134.3, 144.5, 145.6, 146.3, $J=8.0 \mathrm{~Hz}), 11.7\left(\mathrm{~s}, 1 \mathrm{H}, \mathrm{SO}_{2} \mathrm{NH}, \quad 167.3,172.0,180.3,183.0\right.$ $\mathrm{D}_{2} \mathrm{O}$-exchangeable)
$12.1,95.8,100.6,128.4$, $133.7,142.3,145.4,146.0$, $160.3,169.9,180.4$

3.9 (s, 3H, $\left.\mathrm{OCH}_{3}\right), 6.6,7.2$ (2d, 2H, 55.7, 108.1, 113.2, 113.6, $\mathrm{CH}=\mathrm{CH} ; J=8.1,8.3 \mathrm{~Hz}), 7.3-7.5 \quad 121.4,128.8,129.0,136.5$,

136.9, 138.1, 140.2, 142.0, $149.8,165.1,185.8$

$94.9,127.0,127.1,127.2$ $127.3,128.6,129.8,130.2$ $133.1,134.9,143.0,143.1$, $144.8,145.8,146.5,180.2$

23.9, 92.3, 101.9, 122.2, 123.1, 128.2, 128.9, 129.0, $129.5,136.9,138.1,140.2$, 142.0, 142.6, 153.6, 160.2, 185.8

$113.2,115.6$ (2), 128.6 (4), 130.1 (4), 131.0, 133.8, 134.7, $148.8,151.3,152.8,153.9$, $163.4,190.2$ $100.9,112.0,119.6,121.0$, $126.3,128.5$ (2), 129.5, 132.9 (2), 133.3 (2), 133.6, 134.3, $134.5,134.8,141.4,145.7$, $180.0,183.3(2)$ 
M. M. Ghorab et al.: Novel thiophene derivatives with sulfonamide, isoxazole, benzothiazole, quinoline and anthracene moieties as potential anticancer agents, Acta Pharm. 64 (2014) 419-431.

\section{In vitro anticancer activity}

Cytotoxic activity was measured in vitro for the newly synthesized compounds using the SulfoRhodamine-B stain (SRB) assay (35). Cells were plated in 96-multiwell microtiter plates $\left(10^{4}\right.$ cells per well) for $24 \mathrm{~h}$ before treatment with the compound, to allow attachment of cells to the plate wall. Test compounds were dissolved in DMSO and diluted with saline to the appropriate concentration. Different concentrations of the compounds under test (10, 25,50 and $100 \mu \mathrm{mol} \mathrm{L}^{-1}$ ) were added to the cell monolayer. Triplicate wells were prepared for each individual dose. Monolayer cells were incubated with the compound(s) for $48 \mathrm{~h}$ at $37^{\circ} \mathrm{C}$ and in an atmosphere of $5 \% \mathrm{CO}_{2}$. After $48 \mathrm{~h}$, the cells were fixed, washed, and stained for 30 min with $0.4 \%(m / V)$ SRB in $1 \%$ acetic acid. Excess unbound dye was removed by four washes with $1 \%$ acetic acid and attached stain was recovered with Tris-EDTA buffer. Color intensity was measured in an enzyme-linked immunosorbent assay ELISA reader. The relation between the surviving fraction and drug concentration was plotted to get the survival curve for the breast tumor cell line after the specified time. The molar concentration required for $50 \%$ inhibition of cell viability $\left(I C_{50}\right)$ was calculated and the results are given in Table III.

Table III. In vitro anticancer screening of the newly synthesized compounds against the human breast cancer cell line MCF7

\begin{tabular}{|c|c|c|c|c|c|}
\hline \multirow{3}{*}{ Compound } & \multicolumn{4}{|c|}{ Compound concentration $\left(\mu \mathrm{mol} \mathrm{L}^{-1}\right)$} & \multirow{3}{*}{$\begin{array}{c}I C_{50} \\
\left.(\mu \mathrm{mol} \mathrm{L})^{-1}\right)\end{array}$} \\
\hline & 10 & 25 & 50 & 100 & \\
\hline & \multicolumn{4}{|c|}{ Surviving fraction ${ }^{\mathrm{a}}$} & \\
\hline Doxorubicin & $0.551 \pm 0.026$ & $0.480 \pm 0.003$ & $0.139 \pm 0.005$ & $0.130 \pm 0.016$ & 32.00 \\
\hline 2 & $0.814 \pm 0.008$ & $0.660 \pm 0.025$ & $0.419 \pm 0.003$ & $0.393 \pm 0.006$ & 38.25 \\
\hline 3 & $0.792 \pm 0.021$ & $0.701 \pm 0.031$ & $0.347 \pm 0.017$ & $0.378 \pm 0.018$ & 36.24 \\
\hline 4 & $0.807 \pm 0.080$ & $0.723 \pm 0.031$ & $0.293 \pm 0.010$ & $0.133 \pm 0.014$ & 47.30 \\
\hline 5 & $0.845 \pm 0.013$ & $0.515 \pm 0.021$ & $0.380 \pm 0.003$ & $0.443 \pm 0.017$ & 28.85 \\
\hline 6 & $0.541 \pm 0.003$ & $0.323 \pm 0.020$ & $0.360 \pm 0.018$ & $0.460 \pm 0.015$ & 10.25 \\
\hline 7 & $0.480 \pm 0.010$ & $0.327 \pm 0.016$ & $0.313 \pm 0.005$ & $0.381 \pm 0.007$ & 9.70 \\
\hline 8 & $0.693 \pm 0.023$ & $0.503 \pm 0.033$ & $0.377 \pm 0.010$ & $0.391 \pm 0.015$ & 23.48 \\
\hline 9 & $0.443 \pm 0.017$ & $0.251 \pm 0.012$ & $0.355 \pm 0.020$ & $0.290 \pm 0.009$ & 9.55 \\
\hline 10 & $0.810 \pm 0.022$ & $0.550 \pm 0.019$ & $0.331 \pm 0.013$ & $0.350 \pm 0.015$ & 27.51 \\
\hline 11 & $0.872 \pm 0.025$ & $0.638 \pm 0.016$ & $0.370 \pm 0.030$ & $0.307 \pm 0.005$ & 34.50 \\
\hline 12 & $0.825 \pm 0.013$ & $0.668 \pm 0.021$ & $0.307 \pm 0.007$ & $0.271 \pm 0.017$ & 33.55 \\
\hline 13 & $0.435 \pm 0.009$ & $0.233 \pm 0.006$ & $0.371 \pm 0.018$ & $0.309 \pm 0.011$ & 9.39 \\
\hline 14 & $0.792 \pm 0.030$ & $0.361 \pm 0.010$ & $0.145 \pm 0.010$ & $0.174 \pm 0.010$ & 36.40 \\
\hline 15 & $0.659 \pm 0.016$ & $0.569 \pm 0.011$ & $0.137 \pm 0.011$ & $0.233 \pm 0.013$ & 39.70 \\
\hline 16 & $0.920 \pm 0.020$ & $0.417 \pm 0.014$ & $0.272 \pm 0.020$ & $0.146 \pm 0.012$ & 42.50 \\
\hline 17 & $0.904 \pm 0.032$ & $0.685 \pm 0.011$ & $0.355 \pm 0.012$ & $0.209 \pm 0.011$ & 53.01 \\
\hline
\end{tabular}

${ }^{\mathrm{a}}$ Mean \pm S.E, $n=3$. 
M. M. Ghorab et al.: Novel thiophene derivatives with sulfonamide, isoxazole, benzothiazole, quinoline and anthracene moieties as potential anticancer agents, Acta Pharm. 64 (2014) 419-431.

\section{RESULTS AND DISCUSSION}

\section{Chemistry}

The current research deals with the synthesis of a new series of thiophene derivatives with sulfonamide, isoxazole, benzothiazole, quinoline and anthracene moieties through reaction of 2-acetyl thiophene with sulfa-drugs, aromatic amines and heterocyclic aromatic amines, and evaluation of their anticancer activity.

Schemes 1 and 2 display the synthesis of thiophenes having the biologically active sulfonamide 2-11, 3-methylisoxazole 12, 4-methoxybenzo[d] thiazole 13, quinoline 14, 15, benzoylphenylamino 16, and anthracene-9,10-dione 17 moieties.

Enaminone derivatives are highly reactive intermediates extensively used for the synthesis of heterocyclic compounds. Thus, treatment of 3-(dimethylamino)-1-(thiophen-2-yl) prop-2-en-1-one (1) with sulfonamide derivatives in refluxing ethanol/acetic acid mixture afforded the corresponding sulfonamide derivatives 2-11. Structures of the latter products were assigned on the basis of their analytical and spectral data. ${ }^{1} \mathrm{H}$ NMR spectra of compounds 2-11 support the assumption that these structures were in Z-form and not in $E$ form, while the coupling constant of doublet signals for olefinic protons was equal to 7.0$7.8 \mathrm{~Hz}$. Z-form was stabilized by intramolecular hydrogen bonding.

The IR spectra of the reaction products showed in each case three absorption bands corresponding to $2 \mathrm{NH}$ functions in the $3464-3185 \mathrm{~cm}^{-1}$ region, in addition to carbonyl

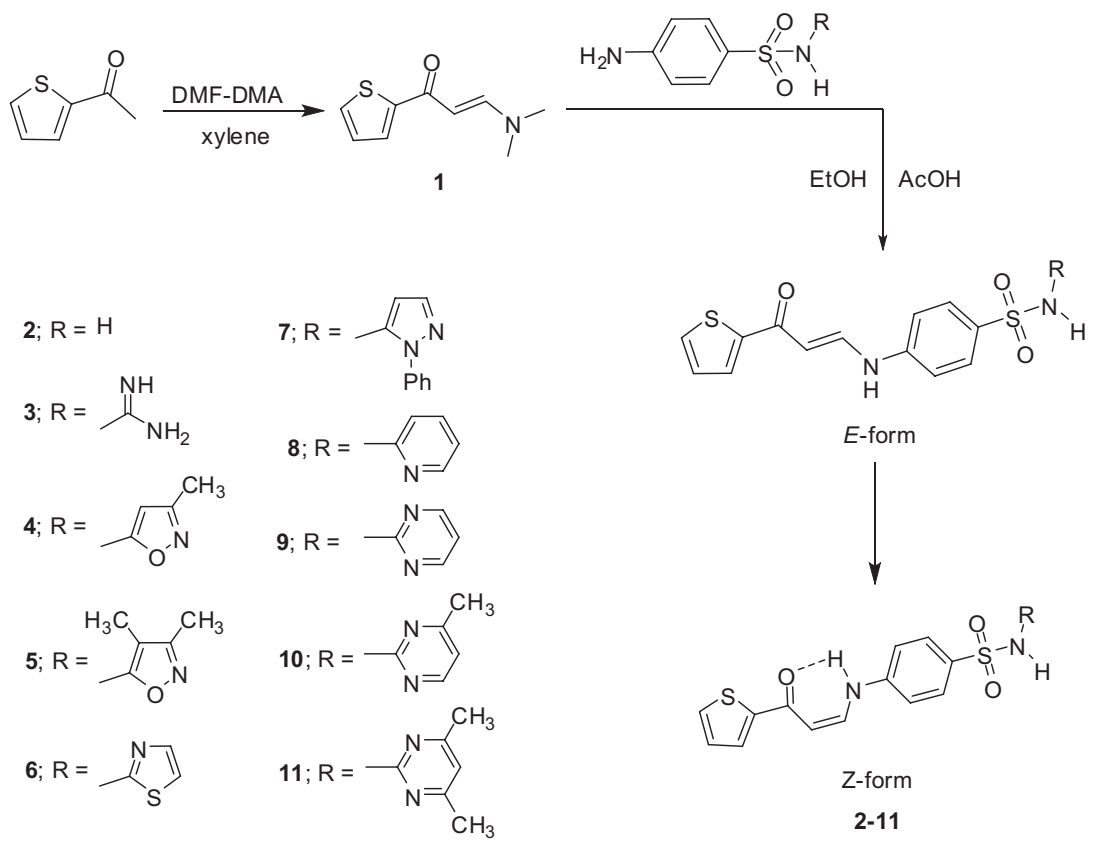

Sheme 1 
absorption band in the 1697-1628 $\mathrm{cm}^{-1}$ region and absorption bands due to $\mathrm{SO}_{2}$ functions in the 1396-1130 $\mathrm{cm}^{-1}$ region. ${ }^{1} \mathrm{H}$ NMR spectra of compounds $\mathbf{2}-\mathbf{1 1}$ revealed a doublet in the 6.1-7.4 ppm region corresponding to the $\mathrm{CH}=\mathrm{CH}$ group.

Also, interaction of compound 1 with 3-methyl-5-aminoisoxazole furnished the corresponding 3-methylisoxazole derivative 12. (Z)-3-(3-Methylisoxazol-5-ylamino)-1-(thiophen-2-yl)prop-2-en-1-one (12) was proven on the basis of elemental analysis and spectral data. Its IR spectrum showed bands at $3132(\mathrm{NH})$ and $1642 \mathrm{~cm}^{-1}(\mathrm{C}=\mathrm{O})$. The ${ }^{1} \mathrm{H}$ NMR spectrum in DMSO- $d_{6}$ revealed signals at $2.3 \mathrm{ppm}$ due to the $\mathrm{CH}_{3}$ group and at $6.4 \mathrm{ppm}$ corresponding to $\mathrm{CH}$ isoxazole. In addition, the corresponding benzothiazole derivative $\mathbf{1 3}$ was obtained in good yield via reaction of 1 with 4 -methoxybenzo[d]thiazol-2-amine. The IR spectrum of 13 exhibited bands at $3421(\mathrm{NH})$ and $1645 \mathrm{~cm}^{-1}(\mathrm{C}=\mathrm{O})$. The ${ }^{1} \mathrm{H}$ NMR spectrum of compound 13 revealed signals at $3.9 \mathrm{ppm}$ attributed to the $\mathrm{OCH}_{3}$ group and at 8.5 ppm due to the NH group. Quinoline derivatives (14 and 15) were obtained via reaction of 1 with quinolin-3-amine or 2-methylquinolin-4-amine, respectively, in a refluxing ethanol/ acetic acid mixture. Structures of compounds 14 and 15 were established on the basis of elemental analysis and spectral data. The IR spectrum of 14 showed bands at $3209 \mathrm{~cm}^{-1}$ $(\mathrm{NH}), 1651 \mathrm{~cm}^{-1}(\mathrm{C}=\mathrm{O})$, and $1612 \mathrm{~cm}^{-1}(\mathrm{C}=\mathrm{N})$. The ${ }^{1} \mathrm{H}$ NMR spectrum of 14 revealed signals at $8.9 \mathrm{ppm}$ due to $\mathrm{N}=\mathrm{CH}$ of quinoline and $11.8 \mathrm{ppm}$ corresponding to the $\mathrm{NH}$ group. The IR spectrum of 15 exhibited bands at $3340 \mathrm{~cm}^{-1}(\mathrm{NH}), 1643 \mathrm{~cm}^{-1}(\mathrm{C}=\mathrm{O})$ and $1620 \mathrm{~cm}^{-1}(\mathrm{C}=\mathrm{N})$. ${ }^{1} \mathrm{H}$ NMR spectrum of 15 revealed signals at $2.4 \mathrm{ppm}$ attributed to the $\mathrm{CH}_{3}$ group and at 8.4 ppm corresponding to the NH group. Interaction of $\mathbf{1}$ with 4-aminophenyl-phenyl-methanone gave the corresponding benzoylphenylamino derivative 16. Its IR spectrum showed bands at $3433(\mathrm{NH})$, and $1640 \mathrm{~cm}^{-1}(2 \mathrm{C}=\mathrm{O})$. The ${ }^{1} \mathrm{H}$ NMR spectrum of 16 showed a multiplet for aromatic protons. Finally, interaction of $\mathbf{1}$ with 2-aminoanthracene-9,10-dione yielded the corresponding anthracene-9,10-dione derivative $\mathbf{1 7}$. Compound $\mathbf{1 7}$ was proven on the basis of elemental analysis and spectral data. Thus, its IR spectrum showed bands at 3444 $\mathrm{cm}^{-1}(\mathrm{NH})$ and $1670,1650 \mathrm{~cm}^{-1}(3 \mathrm{C}=\mathrm{O})$. The ${ }^{1} \mathrm{H}$ NMR spectrum of 17 revealed the presence of a doublet at 6.5 and $6.9 \mathrm{ppm}$ due to $\mathrm{CH}=\mathrm{CH}$ groups, a multiplet at 6.9-8.2 ppm attributed to aromatic protons, and a signal at $10.7 \mathrm{ppm}$ due to the NH group (Scheme 2).

\section{In vitro anticancer activity}

The newly synthesized compounds were evaluated for their in vitro anticancer activity against the human breast cancer cell line MCF7. Doxorubicin was used as the reference drug. Table III shows the in vitro cytotoxic activity of the newly synthesized compounds. Most of the tested compounds exhibited significant activity compared to doxorubicin. It was found that thiophenes containing biologically active sulfathiazole 6 , sulfaphenazole 7 , sulfadiazine 9 , or benzothiazole 13 moieties with respective $I C_{50}$ values of $10.25,9.70,9.55$, and $9.39 \mu \mathrm{mol} \mathrm{L} \mathrm{L}^{-1}$ exhibited 3.1- to 3.4-fold higher anticancer activity than the reference drug with the $I C_{50}$ value of $32.00 \mu \mathrm{mol} \mathrm{L}{ }^{-1}$. Further, thiophenes bearing the biologically active sulfamethoxazole 5 , sulfapyridine 8 and sulfamethazine $\mathbf{1 0}$ with respective $I C_{50}$ values of $28.85,23.48$, and $27.51 \mu \mathrm{mol} \mathrm{L}{ }^{-1}$ were nearly as active as doxorubicin. On the other hand, compounds 2, 3, 11, 12, 14 and 15 revealed slightly lower activity than that of doxorubicin. Thiophene derivatives showed that the cell killing potency against the breast cancer cell line as follows: $13>9>7>6>8>10>5$ doxorubicin. 


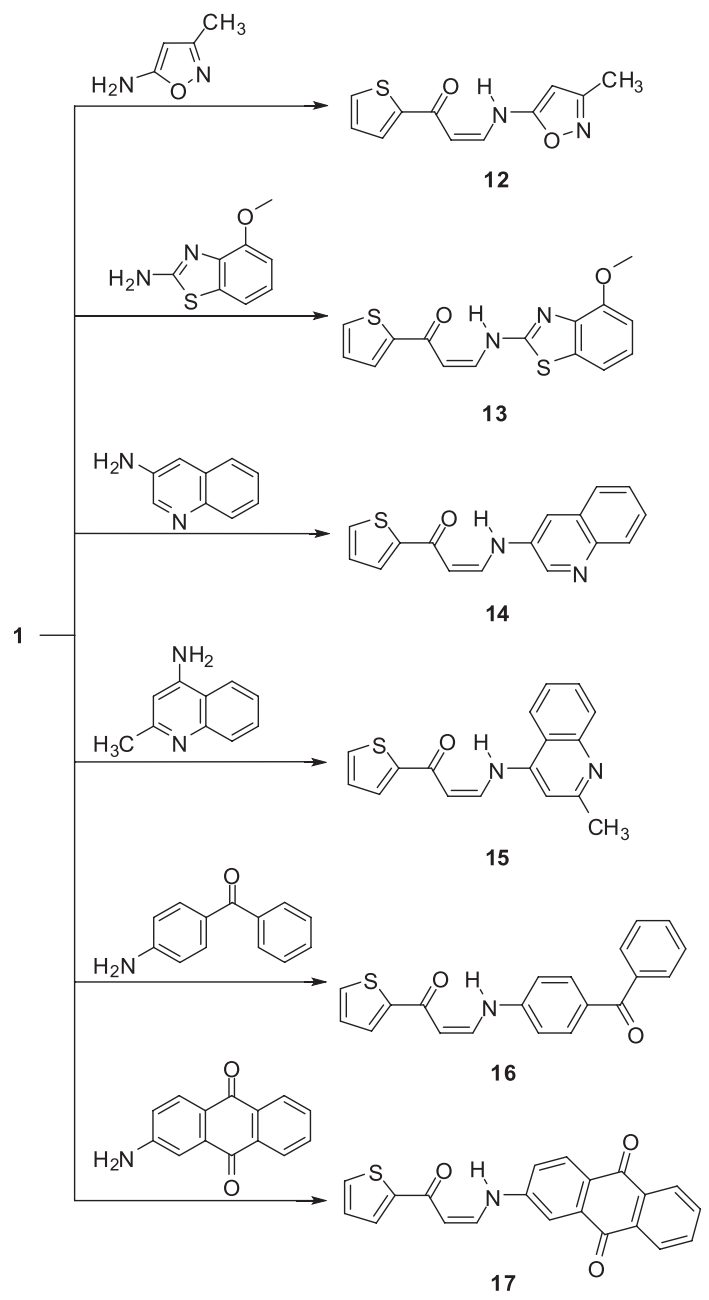

Sheme 2

\section{CONCLUSIONS}

The objective of the present study was to synthesize and investigate the anticancer activity of some novel thiophene derivatives carrying the biologically active sulfonamide, isoxazole, benzothiazole, quinoline and anthracene moieties. Compounds $(Z)-4-(3-0 x o-3-$ (thiophen-2-yl)prop-1-enylamino)- $N$-(thiazol-2-yl)benzenesulfonamide (6), (Z)-4-(3-oxo-3(thio-phen-2-yl)prop-1-enylamino)- $N$-(1-phenyl-1H-pyrazol-5-yl)benzenesulfonamide (7), (Z)-4-(3-oxo-3-(thiophen-2-yl)prop-1-enylamino)- $N$-(pyrimidin-2-yl)benzenesulfonamide (9) and (Z)-3-(4-methoxybenzo[d]thiazol-2-ylamino)-1-(thiophen-2-yl)prop-2-en-1-one (13) showed a promising anti-breast cancer activity, even higher than that of doxorubicin, while compounds (Z)-N-(3,4-dimethyl-isoxazol-5-yl)-4-(3-oxo-3-(thiophen-2-yl)prop-1- 
enylamino)benzene-sulfonamide (5), (Z)-4-(3-oxo-3-(thiophen-2-yl)prop-1-enylamino)- $N$ (pyridin-2-yl)benzenesulfonamide (8) and (Z)-N-(4-methylpyrimidin-2-yl)-4-(3-oxo-3(thiophen-2-yl)prop-1-enylamino)benzenesulfonamide (10) were nearly as active as doxorubicin.

Biological screening of the test compounds could offer an encouraging framework in this field, which may lead to the discovery of even more potent anticancer agents.

Acknowledgements. - The authors extend their sincere appreciation to the Deanship of Scientific Research at King Saud University for its funding of this research through the Research Group Project no. RGP-VPP-302.

\section{REFERENCES}

1. S. Abbas, M. Hussain, S. Ali, M. Parvez, A. Raza, A. Haider and J. Iqbal, Structural, enzyme inhibition, antibacterial and DNA protection studies of organotin(IV) derivatives of thiophene-2carboxylic acid, J. Organometallic Chem. 724 (2013) 255-261; DOI: 10.1016/j.jorganchem.2012.11.033.

2. A. M. Asiri and S. A. Khan, Synthesis and antibacterial activities of a bis-chalcone derived from thiophene and its bis-cyclized products, Molecules 16 (2011) 523-531; DOI: 10.3390/molecules160 10523.

3. A. M. Khalil, M. A. Berghot and M. A. Gouda, Synthesis and antibacterial activity of some new thiazole and thiophene derivatives, Eur. J. Med. Chem. 44 (2009) 4434-4440; DOI: 10.1016/j.ejmech. 2009.06.002.

4. S. Deka, S. Mohan, J. Saravanan, M. Kakati, A. Talukdar, B. J. Sahariah, B. K. Dey and R. K. Sarma, Syntheses, characterization and in-vitro anti-Inflammatory activity of some novel thiophenes, Maced. J. Med. Sci. 5 (2012) 159-163; DOI: org/10.3889/MJMS.1957-5773.2012.0225.

5. R. A. Forsch, J. E. Wright and A. Rosowsky, Synthesis and in vitro antitumor activity of thiophene analogues of 5-chloro-5,8-dideazafolic acid and 2-methyl-2-desamino-5-chloro-5,8-dideazafolic acid, Bioorg. Med. Chem. 10 (2002) 2067-2076; DOI: org/10.1016/S0968-0896(02)00018-4.

6. H. A. Saad, M. M. Youssef and M. A. Mosselhi, Microwave assisted synthesis of some new fused 1,2,4-triazines bearing thiophene moieties with expected pharmacological activity, Molecules, 16 (2011) 4937-4957; DOI: 10.3390/molecules16064937.

7. A. E. Rashad, A. H. Shamroukh, R. E. Abdel-Megeid, A. Mostafa, R. El-Shesheny, A. Kandeil, M. A. Ali and K. Banert, Synthesis and screening of some novel fused thiophene and thienopyrimidine derivatives for anti-avian influenza virus (H5N1) activity, Eur. J. Med. Chem. 45 (2010) 52515257; DOI: 10.1016/j.ejmech.2010.08.044.

8. M. M. Ghorab, F. A. Ragab and M. M. Hamed, Synthesis and docking studies of some novel quinoline derivatives bearing a sulfonamide moiety as possible anticancer agents, Arzneimittelforschung. Drug Res. 60 (2010) 141-148; DOI: 10.1055/s-0031-1296263.

9. M. M. Ghorab, F. A. Ragab, H. I. Heiba and R. M. El-Hazek, Anticancer and radio-sensitizing evaluation of some new thiazolopyrane and thiazolopyranopyrimidine derivatives bearing a sulfonamide moiety, Eur. J. Med. Chem. 46 (2011) 5120-5126; DOI: 10.1016/j.ejmech.2011.08.026.

10. M. M. Ghorab, F. A. Ragab and M. M. Hamed, Design, synthesis and anticancer evaluation of novel tetrahydroquinoline derivatives containing sulfonamide moiety, Eur. J. Med. Chem. 44 (2009) 4211-4217; DOI: 10.1016/j.ejmech.2009.05.017.

11. J. T. Arcari, J.S. Beebe, M. A. Berliner, V. Bernardo, M. Boehm, G. V. Borzillo, T. Clark, B. D. Cohen, R. D. Connell, H. N. Frost, D. A. Gordon, W. M. Hungerford, S. M. Kakar, A. Kanter, N. F. Keene, E. A. Knauth, S. D. LaGreca, Y. Lu, L. Martinez-Alsina and M. A. Marx, Discovery and synthesis of novel 4-aminopyrrolopyrimidine Tie-2 kinase inhibitors for the treatment of solid tumors, Bioorg. Med. Chem. Lett. 23 (2013) 3059-3063; DOI: 10.1016/j.bmcl.2013.03.012. 
12. K. S. Abou-Melha, Octahedral $\mathrm{Co}(\mathrm{II})$ and Ni(II) complexes of schiff bases, semicarbazone and thiosemicarbazone, synthesis, biological, spectral, and thermal studies, J. Coordination Chem. 61 (2008) 2053-2067; DOI: 10.1080/00958970701862167.

13. S. S. Mohamed, S. A. Mohamed, E. S. Shalfoh and O. Fhid, Microwave assisted one-pot synthesis and screening of some schiff's bases of sulfanilamide, J. Chem. Pharmaceutical. Res. 4 (2012) 25122516; DOI: 5-2012/JCPR-2012-4-5-2512-2516.

14. G. D. Simone and C. T. Supuran, Carbonic anhydrase IX: Biochemical and crystallographic characterization of a novel antitumor target, Biochim. Biophys. Acta 1804 (2010) 404-409; DOI: 10.1016/j. bbapap.2009.07.027.

15. S. M. Marques, E. A. Enyedy, C. T. Supuran, N. I. Krupenko, S. A. Krupenko and M. A. Santos, Pteridine-sulfonamide conjugates as dual inhibitors of carbonic anhydrases and dihydrofolate reductase with potential antitumor activity, Bioorg. Med. Chem. 18 (2010) 5081-5089; DOI: 10.1016/j. bmc.2010.05.072.

16. H. M. Said, C. T. Supuran, C. Hageman, A. Staab, B. Polat, A. Katzer, A. Scozzafava, J. Anacker, M. Flentje and D. Vordermark, Modulation of carbonic anhydrase 9 (CA9) in human brain cancer, Curr. Pharm. Des. 16 (2010) 3288-3299; DOI: 10.2174/138161210793429788.

17. W. R. Chegwidden, S. J. Dodgson and I. M. Spencer, The roles of carbonic anhydrase in metabolism, cell growth and cancer in animals, EXS 90 (2000) 343-363; DOI: 10.1016/pubmed.11268523.

18. B. E. Maryanoff and M. J. Costan, Inhibitors of proteases and amide hydrolases that employ an $\alpha$-ketoheterocycle as a key enabling functionality, Bioorg. Med. Chem. 16 (2008) 1562-1595; DOI: 10.1016/j.bmc.2007.11.015

19. S. Bondock, W. Fadaly and M. A. Metwally, Enaminonitrile in heterocyclic synthesis: Synthesis and antimicrobial evaluation of some new pyrazole, isoxazole and pyrimidine derivatives incorporating a benzothiazole moiety, Eur. J. Med. Chem. 44 (2009) 4813-4818; DOI: 10.1016/j.ejmech. 2009.07.024.

20. P. J. Bindu, K. M. Mahadevan and T. R. Ravikumar Naik, An efficient one-pot synthesis and photoinduced DNA cleavage studies of 2-chloro-3-(5-aryl-4,5-dihydroisoxazol-3-yl)quinolines, Bioorg. Med. Chem. Lett. 22 (2012) 6095-6098; DOI: 10.1016/j.bmcl.2012.08.034.

21. M. S. Al-Dosari, M. M. Ghorab, M. S. Alsaid, Y. M. Nissan and A. B. Ahmed, Synthesis and anticancer activity of some novel trifluoromethylquinolines carrying a biologically active benzenesulfonamide moiety, Eur. J. Med. Chem. 69 (2013) 373-383; DOI: 10.1016/j.ejmech.2013.08.048

22. M. Sankaran, C. Kumarasamy, U. Chokkalingam and P. S. Mohan, Synthesis, antioxidant and toxicological study of novel pyrimidoquinoline derivatives from 4-hydroxy-3-acyl quinolin-2one, Bioorg. Med. Chem. Lett. 20 (2010) 7147-7151; DOI: 10.1016/j.bmcl.2010.09.018.

23. A.R. Ellanki, A. Islam, V. S. Rama, R. P. Pulipati, D. Rambabu, G. R. Krishna, M. C. Reddy, K. Mukkanti, G. R. Vanaja, A. M. Kalle, S. K. Kumar and P. Manojit, Solvent effect on copper-catalyzed azide-alkyne cycloaddition (CuAAC): Synthesis of novel triazolyl substituted quinolines as potential anticancer agents, Bioorg. Med. Chem. Lett. 22 (2012) 3455-3459; DOI: 10.1016/j.bmcl.2012. 03.091.

24. L. Wang, M. Switalska, Z. Mei, W. Lu, Y. Takahara, X. Feng, I. E. El-Sayed, J. Wietrzyk and T. Inokuchi, Synthesis and in vitro antiproliferative activity of new 11-aminoalkylamino-substituted $5 \mathrm{H}$ - and $6 \mathrm{H}$-indolo[2,3-b]quinolines; structure-activity relationships of neocryptolepines and 6-methyl congeners, Bioorg. Med. Chem. 20 (2012) 4820-4829; DOI: 10.1016/j.bmc.2012.05.054.

25. L. Fu, W. Lin, P. Xu, Y. Xi, M. Wang and D. Shi, Novel and efficient synthesis of substituted quinoline-1-oxides and the complex compounds $\mathrm{SnL}_{2} \mathrm{Cl}_{2}$ ( $\mathrm{L}=2$-aminoquinoline-1-oxides) with the aid of stannous chloride, Tetrahedron. 68 (2012) 7782-7786; DOI: 10.1016/j.tet.2012.07.049.

26. T. Yamaguchi, S. Watanabe, Y. Matsumura, Y. Tokuoka and A. Yokoyama, Oxovanadium complexes with quinoline and pyridinone ligands: Syntheses of the complexes and effect of alkyl 
chains on their apoptosis-inducing activity in leukemia cells, Bioorg. Med. Chem. 20 (2012) 30583064; DOI: 10.1016/j.bmc.2012.02.063.

27. S. A. F. Rostom, Synthesis and in vitro antitumor evaluation of some indeno[1,2-c]pyrazol(in)es substituted with sulfonamide, sulfonylurea(-thiourea) pharmacophores and some derived thiazole ring systems, Bioorg. Med. Chem. 14 (2006) 6475-6485; DOI: 10.1016/j.bmc.2006.06.020.

28. J. E. Payne, C. Bonnefous, C. A. Hassig, K. T. Symons, X. Guo, P. M. Nguyen, T. Annable, P. L. Wash, T. Z. Hoffman, T. S. Rao, A. K. Shiau, J. W. Malecha, S. A. Noble, J. H. Hager and N. D. Smith, Identification of KD5170: a novel mercaptoketone-based histone deacetylase inhibitor, Bioorg. Med. Chem. Lett. 18 (2008) 6093-6096; DOI: 10.1016/j.bmcl.2008.10.029.

29. M. M. Ghorab, F. A. Ragab, H. I. Heiba, R. K. Arafa and E. M. El-Hossary, Docking study, in vitro anticancer screening and radiosensitizing evaluation of some new fluorine-containing quinoline and pyrimidoquinoline derivatives bearing a sulfonamide moiety, Med. Chem. Res. 20 (2011) 388400; DOI: 10.1007/s00044-010-9332-3.

30. M. S. Alsaid, M. S. Bashandy, S. I. Alqasomi and M. M. Ghorab, Anti-breast cancer activity of some novel 1,2- dihydropyridine, thiophene and thiazole derivatives, Eur. J. Med. Chem. 46 (2011) 137141; DOI: 10.1016/j.ejmech.2010.10.024.

31. M. S. Alsaid, M. S. Bashandy and M. M. Ghorab, Novel quinolines bearing biologically active trimethoxyphenyl moiety as a new class of antitumor agents, Arzneimittel-Forschung Drug Res. 61 (2011) 527-531; DOI: 10.1055/s-0031-1296239.

32. M. S. Bashandy, M. S. Alsaid, S. I. Alqasoumi and M. M. Ghorab, Design and synthesis of some novel hydrazide, 1,2-dihydropyridine, chromene derivative carrying active sulfone moieties with potential anticancer activity, Arzneimittel-Forschung Drug Res. 61 (2011) 521-526; DOI: 10.1055/s0031-1296238.

33. M. S. Alsaid, M. M. Ghorab, M. S. Al-Dosari and M. M. Hamed, Synthesis and in vitro anticancer evaluation of some novel hexahydroquinolinederivatives having a benzenesulfonamide moiety, Eur. J. Med. Chem. 46 (2011) 201-207; DOI: 10.1016/j.ejmech.2010.11.002.

34. F. Al-Omran, N. Al-Awadi, M. M. Abdel-Khalik, K. Kaul, A. A. El-Khair and M. H. Elnagdi,. 1-Substituted 3-dimethylaminoprop-2-en-1-ones as building blocks in heterocyclic synthesis: Routes to 6-aryl- and 6-heteroaryl-2H-pyran-2-ones and 6- and 4-arylpyridin-2(1H)-ones, J. Chem. Res. (S), 84 (1997) 84-85.

35. P. Skehan, R. Storeng, D. Scudiero, A. Monks, J. McMahon, D. Vistica, J. T. Warren, H. Bokesch, S. Kenny and M. R. Boyd, New colorimetric cytotoxicity assay for anticancer-drug screening, J. Natl. Cancer Inst. 82 (1990) 1107-1112; DOI: 10.1093/jnci/82.13.1107. 